\title{
A new approach to linear interval differential equations as a first step toward solving fuzzy differential
}

\author{
Tiago M. da Costa ${ }^{\mathrm{a}, *}$, Yurilev Chalco-Cano ${ }^{\mathrm{b}}$, Weldon A. Lodwick ${ }^{\mathrm{a}}$, Geraldo N. Silva ${ }^{\mathrm{c}}$ \\ ${ }^{a}$ Department of Mathematical and Statistical Sciences, University of Colorado Denver, Denver, CO, 80204, USA \\ ${ }^{\mathrm{b}}$ Instituto de Alta Investigación, Universidad de Tarapacá, Casilla 7-D, Arica, Chile \\ c Department of Applied Mathematics, UNESP - Universidade Estadual Paulista, São José do Rio Preto, Brazil
}

Received 15 December 2015; received in revised form 8 March 2017; accepted 14 October 2017

Available online 18 October 2017

\begin{abstract}
This study uses a concept of interval differentiability, which was recently introduced, to formulate interval initial value problems involving linear interval differential equations. Differently from the approaches that use the $g H$-differentiability, this study does not make use of a criterion of choice for switching points in order to obtain solutions for such problems. The method herein presented provides solutions in a simple, straightforward, and computationally tractable way. Moreover, these solutions are intuitive because they coincide with the solutions given by a differential inclusion method. The efficiency and practicality of our approach are illustrated through some examples that have appeared in other articles.
\end{abstract}

(c) 2017 Elsevier B.V. All rights reserved.

\section{Introduction}

From $[19,21,20]$, it is clear that interval analysis is an important first step in fuzzy interval analysis. Among the topics of fuzzy interval analysis the fuzzy differential equations is one which has a very strong relation with the interval differential equations. One of the motivations for this is that the fuzzy differential equations originally proposed in [24], whose approach is based on the Hukuhara differentiability, have some disadvantages since their solution does not reflect the behavior of the problem initially modeled, see, for example, the radioactivity decay model [13]. In order to provide a model whose solutions describe better the behavior of the problem initially modeled, some articles such as $([14,17])$, present different formalizations to work with fuzzy differential equations. In [18] a new formalization

\footnotetext{
* Corresponding author.

E-mail addresses: grafunjo@yahoo.com.br (T.M.da Costa),ychalco@uta.cl (Y. Chalco-Cano),weldon.lodwick@ucdenver.edu (W.A. Lodwick), gsilva@ibilce.unesp.br (G.N. Silva).
} 
for fuzzy differential equations is presented using the $\alpha$-levels of the fuzzy terms contained in the studied equation. In this sense we consider this work as a first step towards solving fuzzy differential equations since the $\alpha$-levels of a fuzzy number are intervals, and for this reason, we begin with the interval approach.

Differential equation models with interval uncertainties have been studied by many authors, see for example $[13,2-4,8,7,9,16,23,25]$. These articles use differential inclusion methods or the generalized Hukuhara derivative ( $g$ H-derivative, for short).

The behavior of the solutions generated using the concept of the $g H$-differentiability is directly associated with the concept of switching points. The switching points of a $g H$-differentiable interval-valued function are the points where the $g H$-derivatives of such function change from type $(i)$ to type $(i i)$ and vice versa (see $[25,5,6]$ ). Using $g H$-differentiability and setting in an anticipated way that some points are the switching points of a seeked solution for an interval initial value problem, it is possible to construct solutions for periodic boundary value problems involving linear differential equations with uncertainty (see [16]) and, in particular, it is possible to construct solutions for interval periodic differential equations. On the other hand, since these periodic interval solutions are given through a previous choice of switching points, a wrong choice may generate a solution whose behavior does not represent the problem initially modeled. Thus, the difficulty which arises is to know how to determine the best criterion of choice for the switching points in order to construct a reliable solution. The method developed in this study to obtain interval solutions for interval differential equations avoid this kind of mishap since the method provide interval solutions without having to use a criterion of choice for switching points. Each one of these solutions is obtained by solving only one classical linear differential equation in $\mathbb{R}^{2}$. Therefore, the solutions of the interval initial value problems involving linear interval differential equations are obtained in a simple, straightforward, and computationally tractable way.

Unlike the interval differential equations methods involving the $g H$-derivative, to solve interval differential equations via differential inclusion methods allows us to characterize in the interval space the main properties related to ordinary differential equations such as periodicity, stability, bifurcation, among others, in a natural way ([13], [14], [23], [15]). However, the differential inclusion methods do not deal directly with a concept of derivative of intervalvalued functions. That is, the interval differential equations are directly interpreted without having a concept of Interval derivative involved in the process. The method developed in this study uses a concept of interval derivative with which are generated solutions that coincide with the solutions given by a differential inclusion method. Thus our approach allows to interpret the interval differential equations by using directly a interval derivative and also it allows us to generalize in a natural way some properties related to classical differential equations such as those above cited, through such interval derivative.

In short, in this research we study interval initial value problems involving linear interval differential equations by using the concept of $\varphi P$-differentiability of interval-valued functions, which was recently introduced in [11]. This concept of differentiability is equivalent to $g H$-differentiability. However, using the $\varphi P$-differentiable, we obtain solutions for interval initial value problem in a simple, straightforward, and computationally tractable way. Moreover, such solutions coincide with the solutions given by a differential inclusion method.

This research is organized as follows: Section 2 recalls the concepts of limit and of $\varphi$-differentiability of generalized interval-valued functions and some results showing that these concepts are equivalent to the concepts of limit and differentiability of vector-valued functions, respectively. In Section 2 is also recalled the concepts of limit and of $\varphi P$-differentiability of interval-valued functions. The concept of limit of interval-valued functions is obtained by using the concept of limit of generalized interval-valued functions and the $\varphi P$-differentiability is obtained by using the concept of limit of interval-valued functions. Moreover, it is recalled the result which shows that on particular conditions, the existence of $\varphi$-differentiability of a generalized interval-valued function implies the existence of $\varphi P$-differentiability of an interval-valued function and this result plays a key role in the development of our study. Section 2 is finished recalling a result which states that the $\varphi P$-differentiability is equivalent to $\pi$-differentiability, to $g H$-differentiability, and to Markov-differentiability.

Section 3 formalizes the generalized interval initial value problems and through this is also formalized the interval initial value problems. Also in Section 3 is presented the method which allows us to obtain solutions of interval initial value differential equation problems by solving a simple initial value problem in $\mathbb{R}^{2}$. This is one of our main results. Still in the Section 3 is proved that the solutions given by our method coincide with the solutions given by a differential inclusion method, and this is another of our main results. Section 4 presents some numerical examples 
showing in practice the efficiency of the method herein presented. Finally, in the Section 5 final considerations and future directions are given.

\section{Preliminary results and differentiable interval-valued functions}

This section recalls some concepts and the results which are fundamental for the development of this presentation. Most of these concepts and results were given in [11].

This presentation uses $I(\mathbb{R})$ and $\overline{I(\mathbb{R})}$ to denote the family of all proper intervals and the family of all improper intervals given, respectively, by

$$
I(\mathbb{R})=\left\{\left[a_{1}, a_{2}\right]: a_{1}, a_{2} \in \mathbb{R} \text { and } a_{1} \leq a_{2}\right\}
$$

and

$$
\overline{I(\mathbb{R})}=\left\{\left[a_{1}, a_{2}\right]:\left[a_{2}, a_{1}\right] \in I(\mathbb{R})\right\} .
$$

Furthermore, $M:=I(\mathbb{R}) \bigcup \overline{I(\mathbb{R})}$ denotes the set called set of generalized intervals.

Given $\left[a_{1}, a_{2}\right],\left[b_{1}, b_{2}\right] \in M$ we have that $\left[a_{1}, a_{2}\right]=\left[b_{1}, b_{2}\right]$ if and only if $a_{1}=b_{1}$ and $a_{2}=b_{2}$. Lastly, $\varphi: M \longrightarrow \mathbb{R}^{2}$ denotes the bijection defined by

$$
\varphi\left(\left[a_{1}, a_{2}\right]\right)=\left(\lambda_{1} a_{1}+\lambda_{2} a_{2} ; \beta_{1} a_{1}+\beta_{2} a_{2}\right)
$$

with $\lambda_{1}, \lambda_{2}, \beta_{1}, \beta_{2} \in \mathbb{R}$ such that $\lambda_{1} \beta_{2} \neq \lambda_{2} \beta_{1}$, where "+" and "." are the usual algebraic operations in the bidimensional Euclidean vector space. Following [12] $M$ can be equipped with a structure of vector space through the bijection $\varphi$. Thus, the operations $+_{\varphi}$ and $\cdot_{\varphi}$ are defined, respectively, by $\left[a_{1}, a_{2}\right]+_{\varphi}\left[b_{1}, b_{2}\right]=\left[a_{1}+b_{1}, a_{2}+b_{2}\right]$ and $\alpha \cdot \varphi\left[a_{1}, a_{2}\right]=\left[\alpha \cdot a_{1}, \alpha \cdot a_{2}\right]$. In [12] it is showed that (1) is an important class for to solve optimization problems. In this presentation this class of functions (1) is used because it allows us prove some results such as the characterization of derivative of an interval-valued function via derivative of the function extremes of a such interval-valued function, in an easier way since, given arbitrarily $\left[a_{1}, a_{2}\right] \in\left(M,+_{\varphi},{ }_{\varphi}\right)$, its opposite element is given by $\left[-a_{1},-a_{2}\right]$ and, the neutral element with respect to addition $+_{\varphi}$ is the interval $[0,0]$.

From [11], by using the isomorphism $\varphi:\left(M,+_{\varphi}, \cdot_{\varphi}\right) \longrightarrow\left(\mathbb{R}^{2},+, \cdot\right)$ and a norm $\|\cdot\|$ in $\mathbb{R}^{2}$, then $M$ can be equipped with the norm $\|\cdot\|_{\varphi}: M \longrightarrow \mathbb{R}$ defined by $\left\|\left[a_{1}, a_{2}\right]\right\|_{\varphi}:=\left\|\varphi\left(\left[a_{1}, a_{2}\right]\right)\right\|$. Thus the space $\left(M,+_{\varphi}, \cdot_{\varphi},\|\cdot\|_{\varphi}\right)$, which we denote simply by $M_{\varphi}$, it is a normed vector space. The space $M_{\varphi}$ is called generalized interval space, and a map $F: U \subseteq \mathbb{R} \longrightarrow M_{\varphi}$ is called a generalized-interval-valued function.

Given the functions $F, G: U \subseteq \mathbb{R} \longrightarrow M_{\varphi}$ and $\lambda \in \mathbb{R}$, the notation used for algebraic operations between generalized-interval-valued functions are given by $(F+G)(t):=F(t)+{ }_{\varphi} G(t),(\lambda \cdot F)(t):=\lambda \cdot{ }_{\varphi} F(t)$, and $(F-G)(t)=F(t)-{ }_{\varphi} G(t):=F(t)+{ }_{\varphi}(-1) \cdot{ }_{\varphi} G(t)$.

Next we recall other concepts and results introduced in [11].

Definition 2.1. ([11]) Let $F: U \subseteq \mathbb{R} \longrightarrow M_{\varphi}$ be a generalized-interval-valued function. Given $t_{0} \in U$, we say that $L \in M$ is a limit of $F$ as $t \rightarrow t_{0}$ if and only if for every $\epsilon>0$ there exists $\delta>0$ such that

$$
\left\|F(t)-{ }_{\varphi} L\right\|_{\varphi}<\epsilon
$$

for all $t \in U$ with $0<\left\|t-t_{0}\right\|<\delta . L$ is denoted by $\lim _{t \rightarrow t_{0}} F(t)$.

The next result shows that the existence of the limit of a generalized-interval-valued function is equivalent to existence of the limit of a vector-valued function and real variables.

Theorem 2.1.([11]) Let $F: U \subseteq \mathbb{R} \longrightarrow M_{\varphi}$ be a generalized-interval-valued function. Given $t_{0} \in U$, then $L \in M$ is the limit of $F$ as $t \rightarrow t_{0}$ if and only if $L=\varphi^{-1}\left(\lim _{t \rightarrow t_{0}} \varphi(F(t))\right)$.

Definition 2.2. ([11]) Let $F: U \longrightarrow M_{\varphi}$ be a generalized-interval-valued function, where $U \subseteq \mathbb{R}$ is an open set. We say that $F$ is $\varphi$-differentiable at $t \in U$ if and only if there exist the $\operatorname{limit}_{h \rightarrow 0} \frac{1}{h} \cdot \varphi\left(F(t+h)-{ }_{\varphi} F(t)\right)=F_{\varphi}^{\prime}(t)$. The generalized interval $F_{\varphi}^{\prime}(t)$ is called $\varphi$-derivative of $F$ at $t$. 
The next result shows that the existence of the $\varphi$-differentiability of a generalized-interval-valued function is equivalent to existence of the differentiability of a vector function of real variables.

Theorem 2.2. ([11]) Let $F: U \longrightarrow M_{\varphi}$ be a generalized-interval-valued function, where $U \subseteq \mathbb{R}$ is an open set. Then $F$ is $\varphi$-differentiable at $t \in U$ if and only if $H=\varphi \circ F: U \rightarrow \mathbb{R}^{2}$ is differentiable at $t$. Moreover, $F_{\varphi}^{\prime}(t)=\varphi^{-1}\left(H^{\prime}(t)\right)$.

Remark 2.1. Since $\varphi:\left(M,+_{\varphi},{ }_{\varphi}\right) \rightarrow\left(\mathbb{R}^{2},+, \cdot\right)$ is an isomorphism, from definition of $\|\cdot\|_{\varphi}$, it follows that $\varphi: M_{\varphi} \rightarrow\left(\mathbb{R}^{2},+, \cdot,\|\cdot\|\right)$ is a linear homeomorphism. Consequently, $\varphi$ is differentiable and $\varphi^{\prime}(x)=\varphi$ for all $x \in M_{\varphi}$. Thus, considering $F: U \longrightarrow M_{\varphi}$ and $H=\varphi \circ F: U \rightarrow \mathbb{R}^{2}$, from Theorem 2.2, it follows that if $H$ is differentiable at $t$ then $F$ is $\varphi$-differentiable at $t$. Consequently, from the chain rule, it follows that

$$
H^{\prime}(t)=\varphi^{\prime}(F(t)) \circ F_{\varphi}^{\prime}(t)=\varphi\left(F_{\varphi}^{\prime}(t)\right) .
$$

On the other hand, since $H$ is differentiable at $t$, from Theorem 2.2, it follows that

$$
F_{\varphi}^{\prime}(t)=\varphi^{-1}\left(H^{\prime}(t)\right) \text {. }
$$

Therefore (2) can be obtained taking $\varphi$ in the both sides of the equality (3).

The next result shows that the existence of the $\varphi$-differentiability of a generalized-interval-valued function is equivalent to existence of the differentiability of its extremes functions.

Corollary 2.1. ([11]) Let $F: U \longrightarrow M_{\varphi}$ be the generalized-interval-valued function given by $F(t)=\left[f_{1}(t), f_{2}(t)\right]$, where $U \subseteq \mathbb{R}$ is an open set. Then $F$ is $\varphi$-differentiable at $t \in U$ if and only if $f_{1}, f_{2}: U \longrightarrow \mathbb{R}$ are differentiable at $t$. Moreover, $F_{\varphi}^{\prime}(t)=\left[f_{1}^{\prime}(t), f_{2}^{\prime}(t)\right]$.

A map $\hat{F}: U \subseteq \mathbb{R} \longrightarrow I(\mathbb{R}) \subset M_{\varphi}$ is called an interval-valued function. Since $I(\mathbb{R}) \subset M_{\varphi}$, the concept of limit for interval-valued functions can be defined as a particular case of the limit for generalized interval-valued functions. However, the limit of a generalized interval-valued function is an element of $M_{\varphi}$ which does may not belong to $I(\mathbb{R})$. Thus, to obtain a consistent concept of limit for an interval-valued function is required that the limit of an interval-valued function (if exists) be a proper interval.

Definition 2.3. ([11]) Let $\hat{F}: U \subseteq \mathbb{R} \longrightarrow I(\mathbb{R}) \subset M_{\varphi}$ be an interval-valued function. We say that there exist the limit of $\hat{F}$ as $t \rightarrow t_{0} \in U$ if and only if there is $A \in I(\mathbb{R})$ such that $\lim _{t \rightarrow t_{0}} \hat{F}(t)=A$.

Before to present the concept of $\varphi P$-differentiability of interval-valued functions, we need to present the map $p: M \longrightarrow I(\mathbb{R})$ which plays a key role in this presentation. This map is called proper function and given by:

$$
p\left(\left[a_{1}, a_{2}\right]\right)=\left[a_{1}, a_{2}\right]_{p}= \begin{cases}{\left[a_{1}, a_{2}\right],} & \text { if }\left[a_{1}, a_{2}\right] \in I(\mathbb{R}) \\ {\left[a_{2}, a_{1}\right],} & \text { if }\left[a_{1}, a_{2}\right] \in \overline{I(\mathbb{R})} .\end{cases}
$$

Now, using the proper function and the concept of limit of an interval-valued function, we present the concept of $\varphi P$-differentiability of interval-valued functions.

Definition 2.4. ([11]) Let $\hat{F}: U \longrightarrow I(\mathbb{R}) \subset M_{\varphi}$ be an interval-valued function, where $U \subseteq \mathbb{R}$ is an open set. We say that $\hat{F}$ is $\varphi P$-differentiable at $t \in U$ if and only if there is $\hat{F}_{\varphi P}^{\prime}(t) \in I(\mathbb{R})$ such that

$$
\lim _{h \rightarrow 0}\left(\frac{1}{h} \cdot \varphi\left(\hat{F}(t+h)-{ }_{\varphi} \hat{F}(t)\right)\right)_{p}=\hat{F}_{\varphi P}^{\prime}(t)
$$

$\hat{F}_{\varphi P}^{\prime}(t)$ is called the $\varphi P$-derivative of $\hat{F}$ at $t$. 
Through the proper function an interval-valued function can be obtained via a generalized interval-valued function $F: U \subset \mathbb{R} \longrightarrow M_{\varphi}$ by defining the interval-valued function $\hat{F}: U \subset \mathbb{R} \longrightarrow I(\mathbb{R}) \subset M_{\varphi}$ by $\hat{F}(t)=(F(t))_{p}$ for all $t \in U$.

The following result provides a sufficient condition to existence of the $\varphi P$-differentiability of an interval-valued function. Moreover, such result provides a relation between the concepts of $\varphi P$-differentiability and $\varphi$-differentiability.

Theorem 2.3. ([11]) Given an open set $U \subseteq \mathbb{R}$ and a generalized-interval-valued function $F: U \longrightarrow M_{\varphi}$, let $\hat{F}: U \longrightarrow I(\mathbb{R})$ be the interval-valued function given by $\hat{F}(t)=(F(t))_{p}$. If $F$ is $\varphi$-differentiable at $t$, then $\hat{F}$ is $\varphi P$-differentiable at $t$. Moreover, $\hat{F}_{\varphi P}^{\prime}(t)=\left(F_{\varphi}^{\prime}(t)\right)_{p}$.

Example 2.1. Consider the interval-valued function $\hat{F}: \mathbb{R} \longrightarrow I(\mathbb{R})$ defined by $\hat{F}(t)=[\underline{a}, \bar{a}] \cdot g(t)$, where $g: \mathbb{R} \rightarrow \mathbb{R}$ is a differentiable function and $\cdot$ denotes the standard multiplication (see [22]). Then

$$
\hat{F}(t)= \begin{cases}{[\underline{a} g(t), \bar{a} g(t)]} & \text { if } g(t) \geq 0 ; \\ {[\bar{a} g(t), \underline{a} g(t)]} & \text { if } g(t)<0 .\end{cases}
$$

Now, if we consider the generalized-interval-valued function $F: \mathbb{R} \longrightarrow M_{\varphi}$ defined by $F(t)=[\underline{a}, \bar{a}] \cdot \varphi g(t)$, we have $\hat{F}(t)=(F(t))_{p}$ for all $t \in \mathbb{R}$. Since $F$ is $\varphi$-differentiable then, from Theorem 2.3, it follows that $\hat{F}$ is $\varphi P$-differentiable and

$$
\hat{F}_{\varphi P}^{\prime}(t)=\left(F_{\varphi}^{\prime}(t)\right)_{p}=\left(\left[\underline{a} g^{\prime}(t), \bar{a} g^{\prime}(t)\right]\right)_{p}=\left[\min \left\{\underline{a} g^{\prime}(t), \bar{a} g^{\prime}(t)\right\}, \max \left\{\underline{a} g^{\prime}(t), \bar{a} g^{\prime}(t)\right\}\right] .
$$

The interval $\hat{F}_{\varphi P}^{\prime}$ obtained in the last equality coincides with the $g H$-derivative of $F$ at $t$ as can be seen in [10].

The results given by Theorem 2.3 and by Corollary 2.1 allow to obtain a solution for a given interval initial value problem by solving only one classical initial value problem in $\mathbb{R}^{2}$ for which is known solving methods. Moreover, these results allow to avoid a priori choice for the switching points in the solving process.

The next result relates the $\varphi P$-differentiability to $g H$-differentiability.

Theorem 2.4. ([11]) Let $U \subseteq \mathbb{R}$ be an open set. Given $\hat{F}: U \longrightarrow I(\mathbb{R})$ an interval-valued function, $\hat{F}(t)=$ $\left[\hat{f}_{1}(t), \hat{f}_{2}(t)\right]$, then $\hat{F}$ is $\varphi P$-differentiable at $t$ if and only if $\hat{F}$ is $g H$-differentiable at $t$.

The next result provides a necessary and sufficient condition of existence for $\varphi P$-differentiability of an intervalvalued function.

Corollary 2.2. $([8,11])$ Let $U \subseteq \mathbb{R}$ be an open set. Given $\hat{F}: U \longrightarrow I(\mathbb{R})$ an interval-valued function, $\hat{F}(t)=$ $\left[\hat{f}_{1}(t), \hat{f}_{2}(t)\right]$, then $\hat{F}$ is $\varphi P$-differentiable at $t$ if and only if one of the following cases holds

(a) $\hat{f}_{1}$ and $\hat{f}_{2}$ are differentiable at $t$ and

$$
\hat{F}_{\varphi P}^{\prime}(t)=\left[\hat{f}_{1}^{\prime}(t), \hat{f}_{2}^{\prime}(t)\right]_{p}
$$

(b) $\left(\hat{f}_{1}\right)_{-}^{\prime}(t),\left(\hat{f}_{1}\right)_{+}^{\prime}(t),\left(\hat{f}_{2}\right)_{-}^{\prime}(t)$ and $\left(\hat{f}_{2}\right)_{+}^{\prime}(t)$ exist and satisfy $\left(\hat{f}_{1}\right)_{-}^{\prime}(t)=\left(\hat{f}_{2}\right)_{+}^{\prime}(t)$ and $\left(\hat{f}_{1}\right)_{+}^{\prime}(t)=\left(\hat{f}_{2}\right)_{-}^{\prime}(t)$. Moreover,

$$
F^{\prime}(t)=\left[\left(\hat{f}_{1}\right)_{-}^{\prime}(t),\left(\hat{f}_{2}\right)_{-}^{\prime}(t)\right]_{p}=\left[\left(\hat{f}_{1}\right)_{+}^{\prime}(t),\left(\hat{f}_{2}\right)_{+}^{\prime}(t)\right]_{p} .
$$

Since the concepts of $\pi$-differentiability, $g H$-differentiability, and Markov-differentiability are all equivalents (see [8]) and $\varphi P$-differentiability is equivalent to $g H$-differentiability, then $\varphi P$-differentiability is also equivalent to these concepts. 
Remark 2.2. The proper function plays a key role in the development of this study since it helps to define the $\varphi P$-differentiability and it allows to define the class of interval differential equations that we deal in this research. Moreover, using the proper function the Theorem 2.3, which is fundamental for this presentation, is proved.

The next section shows that the use of the $g H$-differentiability in $M_{\varphi}$ (via equivalency with $\varphi P$-differentiability) allows to obtain solutions for interval initial valued problems involving linear interval differential equations in a simple, straightforward, and computationally tractable way, without having to use a criterion of choice for switching points.

\section{Interval initial value problems}

This section presents a method to obtain solutions of interval initial value problems involving linear interval differential equations. Moreover, a relation between the solutions given by this method and the solutions given by a differential inclusion method is presented.

\subsection{Formalization of the generalized interval differential equations and of the interval differential equations}

The formalization of Generalized interval differential equations and the formalization of Interval differential equations is presented here. The Generalized interval differential equations plays a key role in this study since the solutions of an interval initial value problem are obtained by solving a generalized interval initial value problem, which is equivalent to solve a classical initial value problem in $\mathbb{R}^{2}$.

Definition 3.1. Given the functions $Y: U \longrightarrow M$ and $G: U \times M \longrightarrow M$, where $U \subseteq \mathbb{R}$, then the equation

$$
Y_{\varphi}^{\prime}(t)=G(t, Y(t))
$$

is called a generalized interval differential equation.

Definition 3.2. Let $I \subseteq \mathbb{R}$ be an interval. The generalized interval-valued function $\Psi: I \longrightarrow M$ is called a solution of (4) defined on $I$ if and only if $\Psi_{\varphi}^{\prime}(t)=G(t, \Psi(t))$ for all $t \in I$.

A generalized interval linear differential equation (GILDE) is a generalized interval differential equation given by

$$
Y_{\varphi}^{\prime}(t)=a(t) \cdot{ }_{\varphi} Y(t)+{ }_{\varphi} B(t)
$$

where $a: U \subseteq \mathbb{R} \longrightarrow \mathbb{R}$ and $Y, B: U \subseteq \mathbb{R} \longrightarrow M$.

Definition 3.3. Given the functions $X: U \rightarrow I(\mathbb{R})$ and $F: U \times I(\mathbb{R}) \rightarrow I(\mathbb{R})$, where $U \subseteq \mathbb{R}$, then the equation

$$
X_{\varphi P}^{\prime}(t)=F(t, X(t))
$$

is called a interval differential equation.

Definition 3.4. Let $I \subseteq \mathbb{R}$ be an interval. The interval-valued function $\Psi: I \longrightarrow I(\mathbb{R})$ is called a solution of (6) defined on $I$ if and only if $\Psi_{\varphi P}^{\prime}(t)=F(t, \Psi(t))$ for all $t \in I$.

An interval linear differential equation (ILDE) is an interval differential equation given by

$$
X_{\varphi P}^{\prime}(t)=\left(\left(a(t) \cdot{ }_{\varphi} X(t)\right)+{ }_{\varphi} B(t)\right)_{p},
$$

where $a: U \subseteq \mathbb{R} \longrightarrow \mathbb{R}$ and $X, B: U \subseteq \mathbb{R} \longrightarrow I(\mathbb{R})$.

In this work our objects of study are the interval initial value problems involving equations of kind (7). To be more precise, the class of interval initial value problems studied in this presentation is composed by interval initial value problems of kind: 


$$
\left\{\begin{array}{l}
X_{\varphi P}^{\prime}(t)=\left(\left(a(t) \cdot{ }_{\varphi} X(t)\right)+{ }_{\varphi} B(t)\right)_{p} \\
X\left(t_{0}\right)=\left[x_{1}\left(t_{0}\right), x_{2}\left(t_{0}\right)\right]
\end{array}\right.
$$

where $t_{0}, t_{f} \in \mathbb{R}$ with $t_{0} \leq t_{f}$, and $X, B:\left[t_{0}, t_{f}\right] \rightarrow I(\mathbb{R})$, are interval valued functions given, respectively, by $X(t)=\left[x_{1}(t), x_{2}(t)\right]$ and $B(t)=\left[b_{1}(t), b_{2}(t)\right]$, where $a, x_{1}, x_{2}, b_{1}, b_{2}:\left[t_{0}, t_{f}\right] \longrightarrow \mathbb{R}$ are real continuous functions.

Remark 3.1. By using the standard arithmetic (see [22]) and the $g H$-difference (see $[25,5,6]$ ) we have that the right side of (7) is given by:

$$
\left(\left(a(t) \cdot{ }_{\varphi} X(t)\right)+{ }_{\varphi} B(t)\right)_{p}=B(t)+a(t) \cdot X(t), \text { if } 0 \leq a(t),
$$

and

$$
\left(\left(a(t) \cdot{ }_{\varphi} X(t)\right)+{ }_{\varphi} B(t)\right)_{p}=B(t) \ominus(-a(t) \cdot X(t)), \text { if } a(t) \leq 0 .
$$

It is known that many works in the literature such as $[16,25]$ present studies for each one of these cases separately. One of the advantages of using the formalization (7) is that this formalization allows to study both these two cases in a unified way without having to worry about the sign of the function $a(\cdot)$ in a given point $t$.

The method developed in this study consists of providing a solution of (8) solving a generalized interval initial value problem of kind:

$$
\left\{\begin{array}{l}
Y_{\varphi}^{\prime}(t)=c(t) \cdot \cdot_{\varphi} Y(t)+{ }_{\varphi} D(t) \\
Y\left(t_{0}\right)=\left[y_{1}\left(t_{0}\right), y_{2}\left(t_{0}\right)\right]=\left[y_{10}, y_{20}\right]
\end{array}\right.
$$

where $t_{0}, t_{f} \in \mathbb{R}$ with $t_{0} \leq t_{f}, Y:\left[t_{0}, t_{f}\right] \longrightarrow M$ with $Y(t)=\left[y_{1}(t), y_{2}(t)\right]$ and $Y\left(t_{0}\right) \in I(\mathbb{R}), D:\left[t_{0}, t_{f}\right] \longrightarrow I(\mathbb{R})$ with $D(t)=\left[d_{1}(t), d_{2}(t)\right]$, and $c, y_{1}, y_{2}, d_{1}, d_{2}:\left[t_{0}, t_{f}\right] \longrightarrow \mathbb{R}$ are real continuous functions.

\subsection{Solving the Problem (8) via the Problem (9)}

Here, we present a theorem about the existence and uniqueness of solution of (9). We also show that the solution of (9) is a proper interval. Moreover, using the Theorem 2.3 we obtain a solution of (8) by solving the Problem (9).

Theorem 3.1. The Problem (9) has only one solution and this solution is a proper interval.

Proof. From definition of $+_{\varphi}$ and of $\cdot \varphi$, and from Corollary 2.1, it follows that the Problem (9) is equivalent to:

$$
\left\{\begin{array}{l}
y_{1}^{\prime}(t)=c(t) y_{1}(t)+d_{1}(t) \\
y_{2}^{\prime}(t)=c(t) y_{2}(t)+d_{2}(t) \\
y_{1}\left(t_{0}\right)=y_{10} \\
y_{2}\left(t_{0}\right)=y_{20}
\end{array}\right.
$$

From the classical existence and uniqueness theorem, it follows that (10) has only one solution $\left(y_{1}(t), y_{2}(t)\right)$. Consequently, the Problem (9) has only the solution $Y(t)=\left[y_{1}(t), y_{2}(t)\right]$. Moreover, it is known that $\left(y_{1}(t), y_{2}(t)\right)$ is given by

$$
y_{i}(t)=e^{\left(\int_{t_{0}}^{t} c(\tau) d \tau\right)} y_{i}\left(t_{0}\right)+\int_{t_{0}}^{t} e^{\left(\int_{s}^{t} c(\tau) d \tau\right)} d_{i}(s) d s, i=1,2 .
$$

Since $d_{1}(t) \leq d_{2}(t), 0 \leq e^{\left(\int_{s}^{t} c(\tau) d \tau\right)}, 0 \leq e^{\left(\int_{t_{0}}^{t} c(\tau) d \tau\right)} y_{i}\left(t_{0}\right)$ for all $t_{0} \leq s \leq t \leq t_{f}$, and $y_{1}\left(t_{0}\right) \leq y_{2}\left(t_{0}\right)$, then from (11), it follows that $y_{1}(t) \leq y_{2}(t)$ for all $t \in\left[t_{0}, t_{f}\right]$. Therefore, $Y(t)=\left[y_{1}(t), y_{2}(t)\right]$ is a proper interval. 
The next result is one of our main results.

Theorem 3.2. Given the Problem (8), consider the Problem (9) with $Y \equiv X, D \equiv B, d_{1} \equiv b_{1}, d_{2} \equiv b_{2}$, and $a \equiv c$. Then, if $X$ is the solution of (9), it implies that $X$ is a solution of (8).

Proof. Let $X:\left[t_{0}, t_{f}\right] \longrightarrow M$ with $X(t)=\left[x_{1}, x_{2}\right]$ be the solution of (9). That is,

$$
X_{\varphi}^{\prime}(t)=a(t) \cdot{ }_{\varphi} X(t)+{ }_{\varphi} B(t) \text { and } X\left(t_{0}\right)=\left[x_{1}\left(t_{0}\right), x_{2}\left(t_{0}\right)\right] .
$$

Thus, using the proper function we have that

$$
\left(X_{\varphi}^{\prime}(t)\right)_{p}=\left(a(t) \cdot{ }_{\varphi} X(t)+{ }_{\varphi} B(t)\right)_{p} \text { and }\left(X\left(t_{0}\right)\right)_{P}=\left(\left[x_{1}\left(t_{0}\right), x_{2}\left(t_{0}\right)\right]\right)_{p} .
$$

From Theorem 3.1, it follows that $X(t) \in I(\mathbb{R})$ for all $t \in\left[t_{0}, t_{f}\right]$. Consequently, given $\hat{X}:\left[t_{0}, t_{f}\right] \rightarrow I(\mathbb{R})$ by $\hat{X}(t)=(X(t))_{p}$ for all $t \in\left[t_{0}, t_{f}\right]$, it follows that $\hat{X}(t)=X(t)$ for all $t \in\left[t_{0}, t_{f}\right]$, and from Theorem 2.3, it follows that $\left(X_{\varphi}^{\prime}(t)\right)_{p}=X_{\varphi P}^{\prime}(t)$ for all $t \in\left[t_{0}, t_{f}\right]$. Therefore, from (12), it follows that

$$
X_{\varphi P}^{\prime}(t)=\left(a(t) \cdot \cdot_{\varphi} X(t)+{ }_{\varphi} B(t)\right)_{p} \text { and } X\left(t_{0}\right)=\left[x_{1}\left(t_{0}\right), x_{2}\left(t_{0}\right)\right] .
$$

This means that $X$ is a solution of (8).

Remark 3.2. Theorem 3.2 provides our method to obtain solutions for interval initial valued problems involving linear interval differential equations. Our method is simple, straightforward, and computationally tractable because it allows to find a solution for each one of these interval initial valued problems just solving a classical initial value problem in $\mathbb{R}^{2}$. A direct consequence of this fact is that we do not need to make a priori choice for the switching points in order to construct such solutions. Actually, by using our method, the switching points of a solution are given by such solution and not the otherwise.

\subsection{Differential inclusion and interval initial value problem}

Here we provide a theorem showing that the solution of (8) obtained by Theorem 3.2 is more intuitive than the solutions given by the previous approaches which involve treating interval differentiability directly in the solving methods. To be more precise, such result shows that the solution of (8) obtained by Theorem 3.2 coincides with the solution given by the following linear differential inclusion:

$$
x^{\prime}(t) \in\left(a(t) \cdot \varphi x+{ }_{\varphi} B(t)\right)_{p} \text { and } x\left(t_{0}\right) \in X\left(t_{0}\right),
$$

where $x, a:\left[t_{0}, t_{f}\right] \longrightarrow \mathbb{R}, a$ is continuous and $X, B:\left[t_{0}, t_{f}\right] \longrightarrow I(\mathbb{R}) \subset M_{\varphi}$ are given in (8). This solution is considered more intuitive than the solutions given by the previous approaches which involve interval differentiability directly in the solving methods ([13], [14], [23], [15]) because it generalizes in a natural way the properties of solutions of classical differential equations such as periodicity, stability, bifurcation, among others. Mathematically we have that:

Theorem 3.3. Let $X(t)$ be a solution of (8) given by Theorem 3.2. Then $X(t)$ is an attainable set of (13) for all $t \in\left[t_{0}, t_{f}\right]$.

Proof. Consider (13). Since any real number $r$ can be represented as a proper interval $[r, r]$, and the sum of two proper intervals is also a proper interval, then (13) can be rewritten as

$$
x^{\prime}(t) \in a(t) \cdot \varphi[x, x]+{ }_{\varphi} B(t) \text { and } x\left(t_{0}\right) \in X\left(t_{0}\right) .
$$

From definitions of $+_{\varphi}$ and of ${ }_{\varphi}$, it follows that this differential inclusion coincides with the classical linear differential inclusion (see [2]) given by

$$
x^{\prime}(t) \in a(t) \cdot x+B(t) \text { and } x\left(t_{0}\right) \in X\left(t_{0}\right) .
$$

Thus, (13) is equivalent to (14), and consequently, the attainable sets of them also coincide for all $t \in\left[t_{0}, t_{f}\right]$. 
Setting $\Phi(t, s)=e^{\left(\int_{s}^{t} a(\tau) d \tau\right)}$ for all $t, s \in \mathbb{R}$ with $t_{0} \leq s \leq t \leq t_{f}$, since the attainable sets of (13) coincide with the attainable sets of (14), then the attainable set of (13) at $t \in\left[t_{0}, t_{f}\right]$ is given by $R(t)=\Phi\left(t, t_{0}\right) X\left(t_{0}\right)+$ $\int_{t_{0}}^{t} \Phi(t, s) B(s) d s$. Since this integral is the Aumann integral and $\Phi(t, s)>0$, from the definition of the Minkowski algebraic operations, it follows that

$$
\begin{aligned}
R(t)=\left[e^{\left(\int_{t_{0}}^{t} a(\tau) d \tau\right)} x_{x_{1}\left(t_{0}\right)}+\int_{t_{0}}^{t} e^{\left(\int_{s}^{t} a(\tau) d \tau\right)}\right)_{b_{1}(s) d s} \\
\\
e^{\left(\int_{t_{0}}^{t} a(\tau) d \tau\right)_{x_{2}\left(t_{0}\right)+}+\int_{t_{0}}^{t} e^{\left(\int_{s}^{t} a(\tau) d \tau\right)_{\left.b_{2}(s) d s\right]} .}}
\end{aligned}
$$

Therefore, if $X(t)$ is a solution of (8) given by Theorem 3.2, it follows that $X(t)=R(t)$ for all $t \in\left[t_{0}, t_{f}\right]$. That is, $X(t)$ is an attainable set of (13) for all $t \in\left[t_{0}, t_{f}\right]$.

Remark 3.3. It is known that to obtain the attainable sets of a classical differential inclusion, in general, is not an easy task. However, Theorem 3.3 shows that we can obtain the attainable sets of a classical linear one-dimensional linear differential inclusion by solving an interval initial value problem which is an easy task since it means to solve a classical initial value problem in $\mathbb{R}^{2}$.

In short, through Theorem 3.2 we provide solutions for interval initial value problems involving linear interval differential equations in a simple, straightforward, and computationally tractable way which avoids a priori choice for switching points to construct the solution. Moreover, these solutions given by Theorem 3.3 are more intuitive than the solutions given by the previous approaches which involve treating interval differentiability directly in the solving methods.

The next section presents some examples in order to show, in practice, the efficiency of the results introduced here.

\section{Numerical examples}

Here some numerical examples of interval initial value problems (8) are presented using the concepts and results given in the Section 3. We present the graphs of solutions providing a geometric interpretation for them. These examples were studied in previous articles using other methods.

Example 4.1. (Example 40 in [25]): Consider the following interval initial value problem with $t \in[0,4]$

$$
\left\{\begin{array}{l}
X^{\prime}(t)=-X(t)+[1,2] t \\
X(0)=[0,1]
\end{array}\right.
$$

Using (7) we can rewrite (15) as $\left\{\begin{array}{l}X_{\varphi P}^{\prime}(t)=\left(a(t) \cdot{ }_{\varphi} X(t)+{ }_{\varphi} B(t)\right)_{p} \\ X(0)=[0,1],\end{array}\right.$ where $a(t)=-1$ and $B(t)=\left([1,2] \cdot{ }_{\varphi} t\right)_{p}$ for all $t \in[0,4]$. Then, from Theorem 3.2, it follows that the solution $X(t)=\left[x_{1}(t), x_{2}(t)\right]$ is given by the following classical initial value problem 


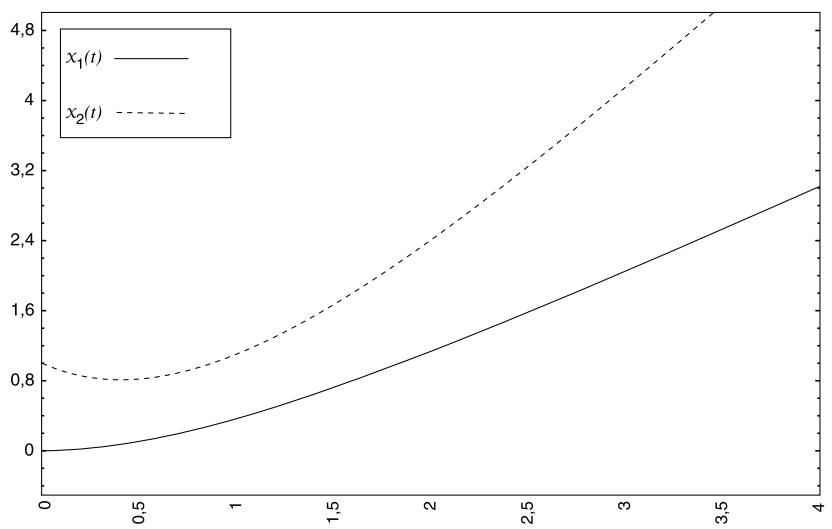

Fig. 1. Solution of (15) given by Theorem 3.2.

$$
\left\{\begin{array}{l}
x_{1}^{\prime}(t)=-x_{1}(t)+t \\
x_{2}^{\prime}(t)=-x_{2}(t)+2 t \\
x_{1}(0)=0 \\
x_{2}(0)=1
\end{array}\right.
$$

whose solution is $\left(x_{1}(t), x_{2}(t)\right)=\left(-1+t+e^{-t},-2+2 t+3 e^{-t}\right)$. Thus, $X(t)=\left[-1+t+e^{-t},-2+2 t+3 e^{-t}\right]$ is a solution of (15). See Fig. 1.

Remark 4.1. The solution $X$ of Example 4.1 does not coincide with any solutions given in Example 40 [25].

Example 4.2. (Example 42 in [25]): Consider the following interval initial value problem with $t \in[0,6]$

$$
\left\{\begin{array}{l}
X^{\prime}(t)=X(t) \sin (t) \\
X(0)=[1,2]
\end{array}\right.
$$

Using (7) we can rewrite (16) as

$$
\left\{\begin{array}{l}
X_{\varphi P}^{\prime}(t)=\left(a(t) \cdot{ }_{\varphi} X(t)+{ }_{\varphi} B(t)\right)_{p} \\
X(0)=[1,2],
\end{array}\right.
$$

where $a(t)=\sin (t)$ and $B(t)=[0,0]$ for all $t \in[0,6]$.

Then, from Theorem 3.2, it follows that the solution $X(t)=\left[x_{1}(t), x_{2}(t)\right]$ is given by the following classical initial value problem

$$
\left\{\begin{array}{l}
x_{1}^{\prime}(t)=x_{1}(t) \sin (t) \\
x_{2}^{\prime}(t)=x_{2}(t) \sin (t) \\
x_{1}(0)=1 \\
x_{2}(0)=2
\end{array}\right.
$$

whose solution is $\left(x_{1}(t), x_{2}(t)\right)=\left(e^{1-\cos (t)}, 2 e^{1-\cos (t)}\right)$. Thus, $X(t)=\left[e^{1-\cos (t)}, 2 e^{1-\cos (t)}\right]$ is a solution of (16). See Fig. 2.

Remark 4.2. The Solution $X$ of Example 4.2 coincides with one of the four solutions given in Example 42 [25].

Example 4.3. (studied in $[13,18,7,1])$ : Consider the following interval initial value problem with $t \in[0,14]$

$$
\left\{\begin{array}{l}
X^{\prime}(t)=-X(t)+[-1,1] \cdot \cos (t) \\
X(0)=[-1,1]
\end{array}\right.
$$




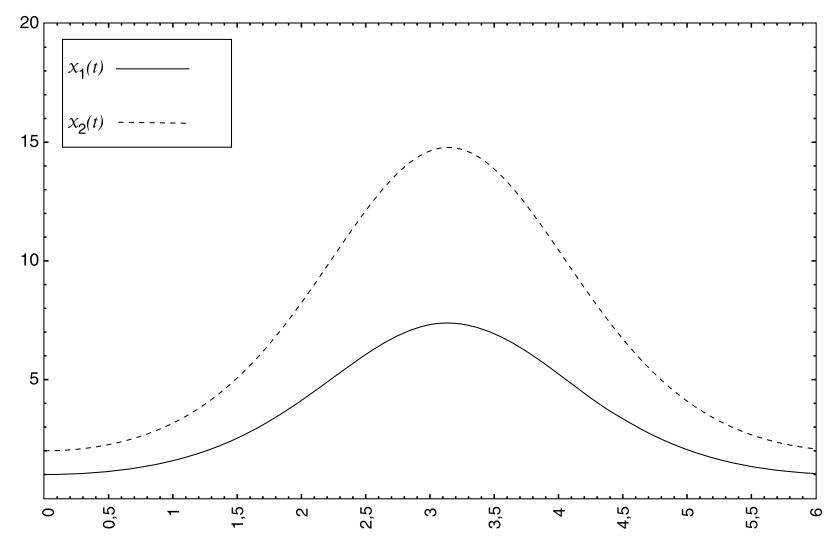

Fig. 2. Solution of (16) given by Theorem 3.2.

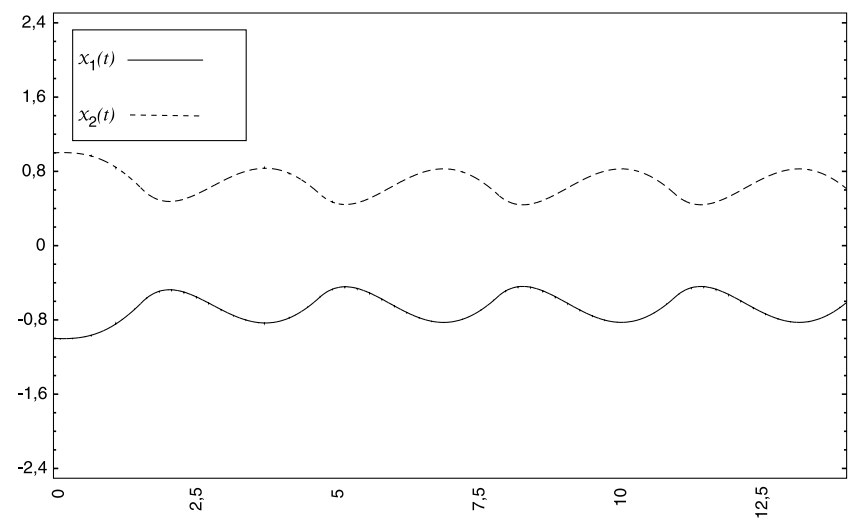

Fig. 3. Solution of (17) given by Theorem 3.2.

Using (7) we can rewrite (17) as

$$
\left\{\begin{array}{l}
X_{\varphi P}^{\prime}(t)=\left(-{ }_{\varphi} X(t)+{ }_{\varphi} B(t)\right)_{p} \\
X(0)=[-1,1]
\end{array}\right.
$$

where $a(t)=-1$ and $B(t)=[-|\cos (t)|,|\cos (t)|]$ for all $t \in[0,14]$. Then, from Theorem 3.2, it follows that the solution $X(t)=\left[x_{1}(t), x_{2}(t)\right]$ is given by the following classical initial value problem

$$
\left\{\begin{array}{l}
x_{1}^{\prime}(t)=-x_{1}(t)-|\cos (t)| \\
x_{2}^{\prime}(t)=-x_{2}(t)+|\cos (t)| \\
x_{1}(0)=-1 \\
x_{2}(0)=1
\end{array}\right.
$$

whose solution is $\left(x_{1}(t), x_{2}(t)\right)=\left(-e^{-t}\left(1+\int_{0}^{t} e^{s}|\cos (s)| d s\right), e^{-t}\left(1+\int_{0}^{t} e^{s}|\cos (s)| d s\right)\right)$. Thus, $X(t)=$ $\left[-e^{-t}\left(1+\int_{0}^{t} e^{s}|\cos (s)| d s\right), e^{-t}\left(1+\int_{0}^{t} e^{s}|\cos (s)| d s\right)\right]$ is a solution of (17). See Fig. 3.

Remark 4.3. The solution $X$ of Example 4.3 coincides with the solution given in Example 1 [7]. 


\section{Conclusion}

By using the concept of $\varphi P$-differentiability, the vector space structure of $\left(M,+{ }_{\varphi}, \cdot_{\varphi}\right)$, and the proper function we provided a new formalization for interval differential equations. Specifically, we studied interval initial value problems involving linear interval equations of kind $X_{\varphi P}^{\prime}(t)=\left(a(t) \cdot{ }_{\varphi} X(t)+{ }_{\varphi} B(t)\right)_{p}$. Differently from some studies presented in the literature such as $[4,8,16,25]$, which work with a linear interval differential equation in a separated way depending on if $a(t)<0$ or if $0 \leq a(t)$, this new formalization allows us to deal with linear interval differential equations in a unified way without having to worry about the sign that the function $a(\cdot)$ attained for each $t$. By using this formalization we obtained from Theorem 3.2 that each interval initial value problem involving linear interval differential equations has, at least, one solution, which can be obtained by solving only one classical initial value problem in $\mathbb{R}^{2}$. Thus, the solution given by Theorem 3.2 is obtained in a simple, straightforward, and computationally tractable way, in which is not necessary a priori choice for switching points to construct such solution. Moreover, from Theorem 3.3 we obtained that the solutions given by Theorem 3.2 coincide with the solutions given by a differential inclusion method. Thus, these solutions generalize properties of solutions of ordinary differential equations such as periodicity, stability, and bifurcation, among others, in a natural way, and this is what makes these solutions be more intuitive than the solutions given by the other approaches that treats interval differentiability directly in the solving process. Theorem 3.3 also shows how to represent the attainable sets of an one-dimensional linear differential equation by solving an interval initial value problem which is interpreted through the concept of $\varphi_{P}$-differentiability.

Our proposal for future work is to use the results presented here and the formalization of fuzzy differential equation given in [17], which uses the $\alpha$-levels of a fuzzy element to solve a fuzzy differential equation. Thus we hope also to obtain a good behavior for the fuzzy differential equations in a simple, straightforward, and computationally tractable way in which will not be necessary to make a priori to choose for switching points in the obtainment process of such solution, even that the model represents a periodic phenomenon. In this sense this research is a first step toward solving fuzzy differential equations.

\section{Acknowledgements}

The authors would like to thank the editors-in-chief, the area editor, and the anonymous referees for their critical comments and helpful suggestions. The first author and the fourth author greatly acknowledge the financial support of the Brazilian National Council for Science and Technology Development (CNPq) under grants numbers 249300/2013-3 and 400754/2014-2, respectively. The second author greatly acknowledges the financial support of FONDECYT under grant number 1151154, the fourth author greatly acknowledges the financial support of the São Paulo State Foundation (FAPESP) grant number 2013/07375-0 through the center for Math. Sciences Applied to Industry-CEPID-CeMEAI.

\section{References}

[1] S. Abbasbandy, J.J. Nieto, M. Alavi, Tuning of reachable set in one dimensional fuzzy differential inclusions, Chaos Solitons Fractals 26 (5) (2005) 1337-1341, https://doi.org/10.1016/j.chaos.2005.03.018.

[2] J.P. Aubin, A. Cellina, Differential Equations of Set-Valued Maps and Viability Theory, Spring-Verlag, Berlin, Heidelberg, New York, Tokyo, 1984.

[3] B. Bede, S. Gal, Generalizations of the differentiability of fuzzy-number-valued functions with applications to fuzzy differential equations, Fuzzy Sets Syst. 151 (3) (2005) 581-599, https://doi.org/10.1016/j.fss.2004.08.001.

[4] B. Bede, I.J. Rudas, A.L. Bencsik, First order linear fuzzy differential equations under generalized differentiability, in: Fuzzy Set Applications in Industrial Engineering, Inf. Sci. 177 (7) (2007) 1648-1662, https://doi.org/10.1016/j.ins.2006.08.021.

[5] B. Bede, L. Stefanini, Solution of fuzzy differential equations with generalized differentiability using LU-parametric representation, in: Proceedings of the 7th Conference of the European Society for Fuzzy Logic and Technology (EUSFLAT-LFA), vol. 1, 2011, pp. 785-790, Advances in Intelligent Systems Research, https://doi.org/10.2991/eusflat.2011.106.

[6] B. Bede, L. Stefanini, Generalized differentiability of fuzzy-valued functions, in: Differential Equations Over Fuzzy Spaces - Theory, Applications, and Algorithms, Fuzzy Sets Syst. 230 (2013) 119-141, https://doi.org/10.1016/j.fss.2012.10.003.

[7] Y. Chalco-Cano, V. de Oliveira, G.N. Silva, Description of the attainable sets of one-dimensional differential inclusions, J. Optim. Theory Appl. 164 (1) (2015) 138-153, https://doi.org/10.1007/s10957-014-0563-1.

[8] Y. Chalco-Cano, H. Román-Flores, M. Jiménez-Gamero, Generalized derivative and $\pi$-derivative for set-valued functions, Inf. Sci. 181 (11) (2011) 2177-2188, https://doi.org/10.1016/j.ins.2011.01.023. 
[9] Y. Chalco-Cano, H. Román-Flores, Some remarks on fuzzy differential equations via differential inclusions, in: Differential Equations Over Fuzzy Spaces - Theory, Applications, and Algorithms, Fuzzy Sets Syst. 230 (2013) 3-20, https://doi.org/10.1016/j.fss.2013.04.017.

[10] Y. Chalco-Cano, A. Rufián-Lizana, H. Román-Flores, M. Jiménez-Gamero, Calculus for interval-valued functions using generalized Hukuhara derivative and applications, in: Theme: Analysis, Fuzzy Sets Syst. 219 (2013) 49-67, https://doi.org/10.1016/j.fss.2012.12.004.

[11] T.M. Costa, W.A. Lodwick, Y. Chaco-Cano, G.N. Silva, A new approach for differentiability of interval-valued functions as first step toward fuzzy differentiability, in: 2015 Annual Conference of the North American Fuzzy Information Processing Society (NAFIPS) held jointly with 2015 5th World Conference on Soft Computing (WConSC), 2015, pp. 1-6, https://doi.org/10.1109/NAFIPS-WConSC.2015.7284176.

[12] T.M. Costa, Y. Chalco-Cano, W.A. Lodwick, G.N. Silva, Generalized interval vector spaces and interval optimization, Inf. Sci. 311 (2015) 74-85, https://doi.org/10.1016/j.ins.2015.03.033.

[13] P. Diamond, Time-dependent differential inclusions, cocycle attractors and fuzzy differential equations, IEEE Trans. Fuzzy Syst. 7 (6) (1999) 734-740, https://doi.org/10.1109/91.811243.

[14] E. Hüllermeier, An approach to modeling and simulation of uncertain dynamical systems, Int. J. Uncertain. Fuzziness Knowl.-Based Syst. 5 (1997) $117-137$.

[15] E. Hüllermeier, Numerical methods for fuzzy initial value problems, Int. J. Uncertain. Fuzziness Knowl.-Based Syst. 7 (5) (1999) 439-461, https://doi.org/10.1142/S0218488599000404.

[16] A. Khastan, J. Nieto, R. Rodríguez-López, Periodic boundary value problems for first-order linear differential equations with uncertainty under generalized differentiability, Inf. Sci. 222 (2013) 544-558, https://doi.org/10.1016/j.ins.2012.07.057, including Special Section on New Trends in Ambient Intelligence and Bio-inspired Systems.

[17] V. Laksmikantham, S. Leela, A. Vatsala, Interconnection between set and fuzzy differential equations, Nonlinear Anal. 54 (2) (2003) 351-360, https://doi.org/10.1016/S0362-546X(03)00067-1.

[18] V. Lakshmikantham, R.N. Mohapatra, Theory of Fuzzy Differential Equations and Inclusions, Taylor \& Francis, New York, 2003.

[19] W.A. Lodwick, Interval and fuzzy analysis: a unified approach, in: Special Issue Celebrating the 50th Anniversary of Fuzzy Sets, Adv. Imaging Electron Phys. 148 (2015) 75-192, https://doi.org/10.1016/S1076-5670(07)48002-8.

[20] W.A. Lodwick, D. Dubois, Interval linear systems as a necessary step in fuzzy linear systems, in: Special Issue Celebrating the 50th Anniversary of Fuzzy Sets, Fuzzy Sets Syst. 281 (2015) 227-251, https://doi.org/10.1016/j.fss.2015.03.018.

[21] W.A. Lodwick, O. Jenkins, Constrained intervals and interval spaces, Soft Comput. 17 (8) (2013) 1393-1402, https://doi.org/10.1007/ s00500-013-1006-X.

[22] R.E. Moore, Interval Analysis, Prentice-Hall, Englewood Cliffs, NJ, 1966.

[23] N.V. Plotnikova, Systems of linear differential equations $\pi$-derivative and linear differential inclusions, Sb. Math. 196 (11) (2005) $1677-1691$.

[24] M.L. Puri, D.A. Ralescu, Differentials of fuzzy functions, J. Math. Anal. Appl. 91 (2) (1983) 552-558, https://doi.org/10.1016/ 0022-247X(83)90169-5.

[25] L. Stefanini, B. Bede, Generalized Hukuhara differentiability of interval-valued functions and interval differential equations, Nonlinear Anal. 71 (34) (2009) 1311-1328, https://doi.org/10.1016/j.na.2008.12.005. 